\title{
Energetic Particle Tracing of Interplanetary CMEs: ULYSSES/HI-SCALE and ACE/EPAM Results
}

\author{
Olga E. Malandraki ${ }^{1} \dagger$, D. Lario ${ }^{2}$, T.E. Sarris ${ }^{1}$, N. Tsaggas ${ }^{1}$ \\ and E.T. Sarris ${ }^{1}$ \\ ${ }^{1}$ Democritus University of Thrace, Space Research Laboratory, Xanthi, 67100 Greece, \\ email: omaland@xan.duth.gr, sarris@ee.duth.gr \\ ${ }^{2}$ Applied Physics Laboratory, the Johns Hopkins University, Laurel, Maryland, USA \\ email: david.lario@jhuapl.edu
}

\begin{abstract}
Solar energetic particle (SEP) fluxes measured by the ULYSSES (ULS)/HI-SCALE experiment during its second polar orbit as well as by the identical ACE/EPAM experiment at $1 \mathrm{AU}$ are utilized as diagnostics of the large-scale structure and topology of the Interplanetary Magnetic Field (IMF) embedded within Interplanetary Coronal Mass Ejections (ICMEs). Survey results are also reported.
\end{abstract}

Keywords. Sun: coronal mass ejections (CMEs), interplanetary medium, magnetic fields

\section{Introduction}

First estimates of the spatial extent of magnetic loops to distances $\sim 3.5$ AU from the Sun were obtained by Sarris \& Krimigis 1982. In this paper, we use SEP measurements by the ULS/HI-SCALE (Lanzerotti, Gold, Anderson et al. (1992)) and ACE/EPAM experiments (Gold, Krimigis, Hawkins et al. (1998)) in order to trace the topology of ICMEs. We present the first observations of a high-latitude ICME that involves complex intertwined structures including regions both connected to and disconnected from the Sun. Results of an ICME survey are also presented.

\section{Observations \& Data Analysis}

In figure 1, ion, electron $\left(e^{-}\right)$, solar wind and magnetic field observations by the EPAM, SWEPAM and MAG experiments onboard ACE are presented from October 29-31 (DOY 302-304), 2003. Two of the fastest ICMEs ever measured in the solar wind were detected during this period and in early November 2003. These very fast ICMEs drove shocks identified in the ACE magnetic field data (solid lines S1 and S2 in figure 1). B was particularly enhanced following the S1 shock, briefly reaching $68 \mathrm{nT}$. The maximum speed on Oct 29th was $1900 \mathrm{~km} / \mathrm{sec}$; the largest speed associated with the second ejection, late Oct 30th, was $1940 \mathrm{~km} / \mathrm{sec}$. The first ICME is associated with an X17.2/4B (S16 E08) solar flare which started at 0951 on Oct 28 and an associated halo CME at 1130 on Oct 28 whereas the 2nd ICME is associated with an X10.0/2B (S15 W02) solar flare which started at 2037 UT on Oct 29 and an associated halo CME which first appeared in the $\mathrm{C} 2$ coronagraph of the SOHO/LASCO experiment at $2054 \mathrm{UT}$ on Oct 29 (http://cdaw.gsfc.nasa.gov/).

We argue that the two dashed lines in figure 1 consist a more accurate determination of the boundaries of the 1st ICME. Smooth rotations of the B direction start to be observed

$\dagger$ Also at National Observatory of Athens, Inst. for Space Applications, Athens, 15236, Greece 
at the 1st dashed line. Furthermore, at that time, there is a change in B from high to low variance in both magnitude and direction, which is a typical $B$ signature of ejecta, and a decrease in the $\sim \mathrm{MeV}$ ion intensity. An abrupt increase at the low-energy ion intensity is observed at the 2 nd dashed line. In ecliptic observations at 1 AU have shown that during SEP events, the particle intensity level is usually higher outside the ejecta. Low-energy ion intensities usually peak at the arrival of interplanetary (IP) shocks driven by fast CMEs (Cane et al. (1988)). When the spacecraft enters into the ejecta, there is often a decrease in the ion intensities with respect to the intensities observed around the arrival of the shock (Richardson 1997), often followed by a recovery of the particle intensity at/near the exit from the ejecta. At the 2nd dashed line a change in the particle Pitch Angle Distributions (PADs) from bi-directional (BD) to isotropic is also observed (see below) along with an abrupt change in the azimuthal angle of the field.

A large gradual $e^{-}$event is observed within this ICME (figure 1) superposed upon the decay phase of a previous $e^{-}$event associated with the October 28 solar events. Large gradual SEP events at $1 \mathrm{AU}$ are associated with particle acceleration at CME-driven coronal and IP shocks (Kahler, Cane, Hudson et al. (1998), Reames 1999). The $e^{-}$fluxes are observed to rise simultaneously at all energies at 2145 UT on Oct 29. The high preevent ambient intensities mask the onset of this $e^{-}$event. PADs with a much stronger $\mathrm{BD}$ character, start to be detected at the time of the $e^{-}$enhancement. BD PADs with variable anisotropy magnitudes were observed till the trailing edge of this ICME was convected over the s/c when the $e^{-}$PADs switch to isotropic.
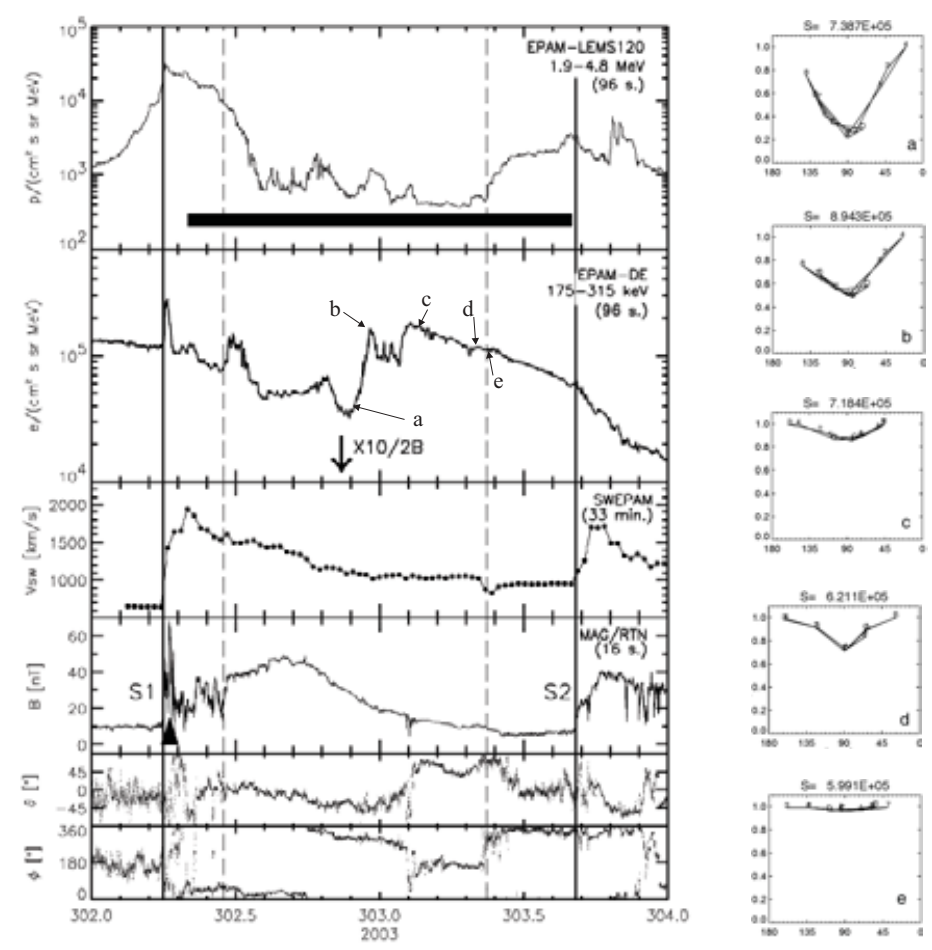

Figure 1. Ion, electron, solar wind and magnetic field observations by the EPAM, SWEPAM and MAG experiments onboard ACE from October 29-31 (DOY 302-304), 2003. The black horizontal bar indicates the time interval identified by Skoug, Gosling, Steinberg et al. (2004) as the 1st ICME. 

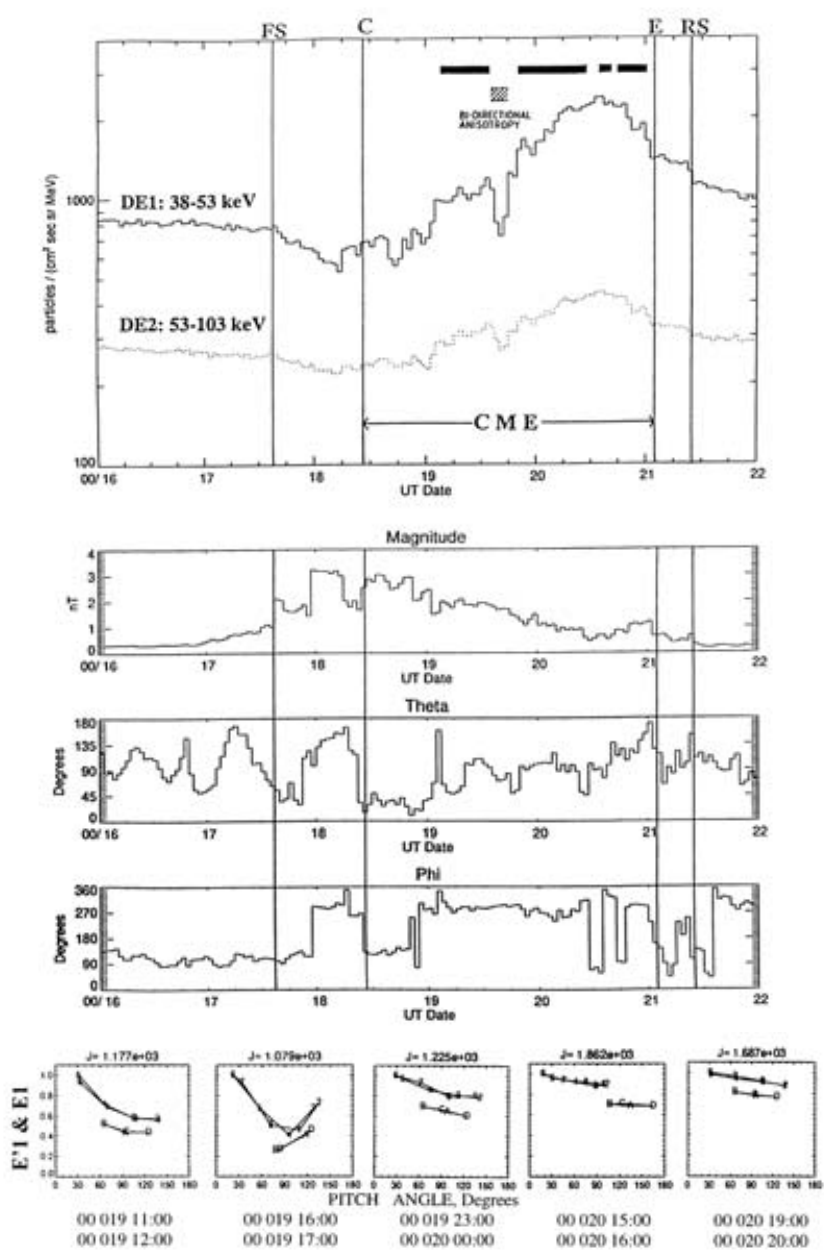

Figure 2. Vertical traces $\mathrm{C}$ and $\mathrm{E}$ denote the Commencement and End of the high latitude ICME observed by ULS. Electron PAD snapshots are presented at the bottom.

In figure 2, an overview of the hourly and spin-averaged differential intensities of $e^{-}$in two energy channels as measured by the ULS/HI-SCALE experiment from 16-22 January, 2000 is presented. IMF parameters in the heliocentric RTN system are also shown. An ICME bounded by the vertical traces $\mathrm{C}$ and $\mathrm{E}$ was detected by ULS on its way towards the Sun's south pole (Gosling \& Forsyth 2001). At this time, ULS was located at $43^{\circ} \mathrm{S}$, at $4.1 \mathrm{AU}$ helioradius and $48^{\circ}$ west of the Earth. Using the solar wind velocity of 400 $\mathrm{km} / \mathrm{sec}$ measured by the SWOOPS experiment on Jan 19, the Parker spiral magnetic field line connecting ULS to the Sun, was computed to be 7.84 AU long. The ULS nominal magnetic connection longitude was at E60 on the visible hemisphere of the Sun.

A distinct enhancement is observed at energies $>38 \mathrm{keV}$ at $0105 \mathrm{UT}$ on Jan 19. Since the transit time for $38-53 \mathrm{keV} e^{-}$with a $0^{\circ}$ pitch angle along the spiral is 2.5 hours, an initial injection at the Sun at 2235 UT on Jan 18 would be implied. There were no significant Ha or X-ray flares reported in SGD, 2000. However, numerous IP type III radio bursts were captured by the WIND/WAVES instrument around the anticipated injection 
time and during Jan 19. The event has a 1.5 day risetime to maximum. The slow risetime of the event, the absence of a velocity dispersion and the non-impulsive nature of the rising phase indicate the $e^{-}$detected after their injection must have undergone drifts across the highly inhomogeneous magnetic fields near the Sun before reaching the ULS footpoint and escaping in space, populating this high latitude ICME.

PADs reveal that after the onset of the event, ULS enters a region where strong unidirectional flow is observed (figure 2). The anisotropy is directed parallel to the IMF, indicating the detected $e^{-}$comprise an $e^{-}$beam streaming away from the Sun. Unidirectional PADs persist till 1500 UT on Jan 19 (horizontal solid bars in figure 2) when PADs dramatically change from unidirectional to strongly BD (hatched horizontal bar in figure 2). This transition coincides with an abrupt decrease observed in the flux profile of the event. ULS remained in this region for $3 \mathrm{hrs}$ and upon exiting the fluxes recovered their values. After a 2-hr interval of isotropy, ULS encounters a regime where $e^{-}$are again streaming antisunward for $15 \mathrm{hrs}$. PAD snapshots are also shown for the regions observed near the rear boundary of the ICME with $e^{-}$streaming away from the Sun.

\section{Discussion}

The observation of an SEP event within the the October 29-31, 2003 ICME implies that the field lines threading through this structure are still rooted at the Sun allowing direct access of $e^{-}$. The BD anisotropies within the ICME are signatures of strong trapping of the observed $e^{-}$population. Assuming the ICME is an open structure, when ACE is inside the ICME it connects directly to the S1 shock (beyond the observer) and it also connects to the $\mathrm{S} 2$ shock which is coming from the Sun. The enhanced B region reaching $\sim 68 n T$ and lying downstream from the preceding S1 shock passes ACE at $\sim 0600$ on Oct 29 and is travelling radially away the Sun with $1900 \mathrm{~km} / \mathrm{sec}$ (figure 1). At the time of the acceleration and injection of the energetic $e^{-}$close to the Sun i.e. 2037 UT this compressed B region is located at a 0.6 AU radial distance upstream from ACE. In such an open magnetic field configuration, observed energetic $e^{-}$streaming in an antisunward direction, after having been reflected at this magnetic constriction in space are detected at the time of the $e^{-}$enhancement observed at 2145 UT. Subsequently, reflection from the following S2 shock of these $e^{-}$propagating sunward results to particle trapping within the region between the two shocks. Another configuration consistent with the observations is a closed magnetic field topology. When the observer is inside the ICME there is no direct connection between the observer and S1 (now beyond the observer). Hence, it is easy to explain the depression of the energetic ions observed at the leading edge of the ICME. The BD PADs in this scenario could result from $e^{-}$injected from both sides of connection of a loop-like structure to S2. Particles are trapped within this configuration by reflection from $\mathrm{S} 2$, bouncing back and forth between the legs of the looped B lines. A fraction of particles moving from the weak field side into the strong field are reflected by the shock magnetic field discontinuities. $E^{-}$within the loss cone can leak from the trapping region. The evolution of the particle flux is determined by the balance between the continuous particle injection from the following shock into the storage region and the loss of particles passing through the shock(s).

Within the high latitude ICME we have observed energetic $e^{-}$to be streaming for long intervals in a collimated beam along IMF lines threading through the ICME. Therefore, a large portion of the ICME most likely consisted of open IMF filaments connected to the Sun at only one end that allowed the escape of $e^{-}$to $4.1 \mathrm{AU}$ and 43S. However, the $3 \mathrm{hr}$ long abrupt depression observed in the flux profiles that interrupted the event risephase indicates that injected particles at the Sun could not enter this ICME portion. 
We conclude that the IMF within this portion of the ICME is most likely disconnected from the Sun (Malandraki, Sarris, Lanzerotti et al. (2001). Furthermore, the observation of counterstreaming $e^{-}$throughout this region implies that the $e^{-}$are propagating within closed looped IMF lines in space. The $e^{-}$observed within this detached looped region may have been imitially injected at the Sun before disconnection occurred. If $e^{-}$are injected from the Sun while a plasmoid (a closed magnetically isolated structure) is in space, these $e^{-}$would be excluded from the plasmoid since they would have to diffuse across IMF lines to enter it. Such regions moving over ULS would be accompanied by substantial reductions in $e^{-}$fluxes, in agreement with what is observed.

We have performed a survey of ICMEs observed by ULS and ACE in different regions of the heliosphere and found that the large majority of the ICMEs $(\sim 92 \%)$ are still magnetically anchored to the Sun when they arrive at the s/c whereas for only $\sim 5 \%$ of the ICMEs there is strong observational evidence that closed magnetic loop-like structures detached from the Sun are present (Malandraki (2002)). Our particle observations suggest that in rare cases ICMEs involve more complex intertwined structures including regions both connected to and disconnected from the Sun. An open magnetic field line topology with open magnetic field lines rooted to the Sun at only one end threading through the ICMEs tends to be the prevalent magnetic structure of ICMEs (e.g. Malandraki, Sarris, Lanzerotti et al. (2002), Malandraki, Sarris, Trochoutsos et al. (2004)). However, the detection of trapped energetic $e^{-}$within a number of ICMEs $(\sim 9 \%)$ is consistent with the presence of closed loop-like magnetic structures anchored to the Sun at both ends.

\section{Acknowledgements}

We are thankful to our HI-SCALE team colleagues for their support and encouragement. Olga Malandraki acknowledges support from the State Scholarships Foundation (I.K.Y.) through a Post-Doctoral Fellowship.

\section{References}

Cane, H.V., Reames, D.V. \& von Rosenvinge, T.T. 1988, J. Geophys. Res. 93, 9555

Gold, R.E., Krimigis, S.M., Hawkins S.E, Haggerty, D.K., Lohr, D.A., Fiore, E., Armstrong, T.P., Holland, G., Lanzerotti, L.J. 1998, Space Sci. Rev. 86, 541

Gosling, J.T. \& Forsyth, R.J. 2001, Space Sci. Rev. 97, 263

Kahler, S.W., Cane, H.V., Hudson, H.S., Kurt, V.G., Gotselyuk, Y.V., MacDowall, R.J., Bothmer, V., 1998, J. Geophys. Res. 103, 12069

Lanzerotti, L.J., Gold. R.E., Anderson, K.A., Armstrong, T.P., Lin, R.P., Krimigis, S.M., Pick, M., Roelof, E.C., Sarris, E.T., Simnett, G.M., Frain, W.E. 1992, Astron. Astrophys. Suppl. Ser. 92, 349

Malandraki, O.E., Sarris, E.T., Lanzerotti, L.J., Maclennan, C.G., Pick, M., Tsiropoula, G. 2001, Space Sci. Rev. 97, 263

Malandraki, O.E. 2002, Ph.D. thesis, Democritus University of Thrace, Xanthi, Greece

Malandraki, O.E., Sarris, E.T., Lanzerotti, L.J., Trochoutsos, P., Tsiropoula, G., Pick, M. 2002 J. Atmos. Sol.-Ter. Phys. 64/5-6, 517

Malandraki, O.E., Sarris, E.T., Trochoutsos, P., Tsiropoula, G., 2004 in: P.G. Laskarides (ed.), Proceedings of the 6th Hellenic Astronomical Society Conference (Athens, Greece) p. 51

Reames, D.V. 1999, Space Sci. Rev. 90, 413

Richardson, I.G. 1997 in: N. Crooker, J.A. Joselyn, and J. Feynman (eds.), Coronal Mass Ejections, Geophys. Monogr. Ser. (AGU, Washington D.C.), vol. 99, p. 189

Sarris, E.T. \& Krimigis, S.M. 1982, .Geophys. Res. Lett. 9, 167

Skoug, R.M., Gosling, J.T., Steinberg, J.T., McComas, D.J., Smith, C.W., Ness, N.F., Hu, Q., Burlaga, L.F. 2004 J. Geophys. Res. 109, A09102, doi:10.1029/2004JA010494 


\section{Discussion}

KAHLER: I am confused by seeing the interpretation of a plasmoid imbedded inside a unidirectional field, which you gave (I think) to your last event.

MALANDRAKI: We think it's the most plausible interpretation of the observations. The flux depression observed for $3 \mathrm{hrs}$ signifies the electrons cannot enter this portion of the ICME, implying it is probably disconnected from the sun. Moreover, the observations of a dramatic change from unidirectional to bidirectional PADS throughout this ICME region is consistent with the electrons propagating within closed looped IMF lines in space.

Kouтснмy: Regarding those beams of energetic electrons related to type III bursts of very short duration, what are their typical durations?

MALANDRAKI: By stating that we have observed type III burst activity associated with the electron beams detected by Ulysses/HI-SCALE, I meant a type III burst storm was observed by WIND/WAVES ie., an almost continuous succession of type III bursts for $\sim 1.5$ day.

BothMER: The interpretation of suprathermal electrons and energetic particles is opposite previous interpretations where BDEs are signatures of closed fields throughout CME. Energetic articles may be indicative of connection to the Sun rather than of closed/open structure.

MALANDRAKI: In our work, we use Near-Relativistic electrons (38-315kev) measured by the Ulysses/HI-SCALE and ACE/EPAM experiments to trace the large-scale structure of Interplanetary Coronal Mass Ejections (ICMEs). Observations of bi-directional Pitch Angle Distributions (PADs) are consistent with a loop-like structure connected to the Sun at both ends. Furthermore, open field lines forming a magnetic constriction in space upstream from the S/C can reflect the outgoing electrons and produce the observed counterstreaming electrons. In some cases, from the time delay between the first outgoing and the first back-streaming electrons (provided by the measurement capacities of HI-SCALE and EPAM) it is possible to distinguish between the 2 topologies (see eg. Malandraki et al., J. Atmos. Sol-Terr. Phys., 64/5-6, 517, 2002). 